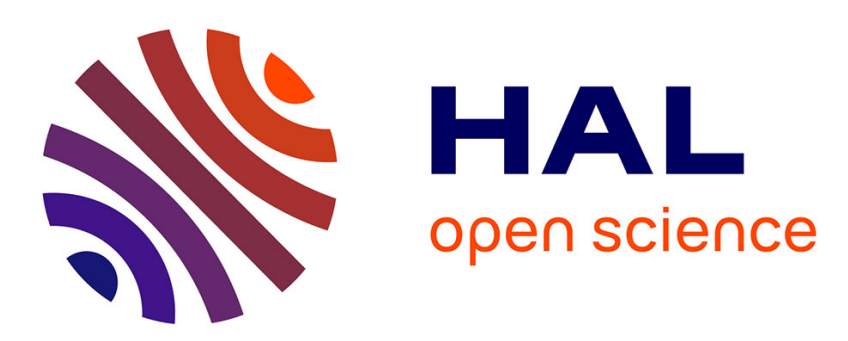

\title{
Caractérisation des cellules solaires silicium (n)-In2O3 (dope Sn) préparées par une méthode de vaporisation
}

\author{
J. Calderer, J.-C. Manifacier, L. Szepessy, J.-M. Darolles, M. Perotin
}

\section{To cite this version:}

J. Calderer, J.-C. Manifacier, L. Szepessy, J.-M. Darolles, M. Perotin. Caractérisation des cellules solaires silicium (n)-In2O3 (dope Sn) préparées par une méthode de vaporisation. Revue de Physique Appliquée, 1979, 14 (3), pp.485-490. 10.1051/rphysap:01979001403048500 . jpa-00244619

\section{HAL Id: jpa-00244619 https://hal.science/jpa-00244619}

Submitted on 1 Jan 1979

HAL is a multi-disciplinary open access archive for the deposit and dissemination of scientific research documents, whether they are published or not. The documents may come from teaching and research institutions in France or abroad, or from public or private research centers.
L'archive ouverte pluridisciplinaire HAL, est destinée au dépôt et à la diffusion de documents scientifiques de niveau recherche, publiés ou non, émanant des établissements d'enseignement et de recherche français ou étrangers, des laboratoires publics ou privés. 


\title{
Caractérisation des cellules solaires silicium (n)- $\operatorname{In}_{2} \mathbf{O}_{3}$ (dope Sn) préparées par une méthode de vaporisation
}

\author{
J. Calderer. J. C. Manifacier, L. Szepessy, J. M. Darolles et M. Perotin \\ Centre d'Etudes d'Electronique des Solides $\left({ }^{*}\right)$, \\ Université des Sciences et Techniques du Languedoc, 34060 Montpellier Cedex, France.
}

(Reçu le 5 juillet 1978, révisé le 27 novembre 1978, accepté le 1er décembre 1978)

\begin{abstract}
Résumé. - La cellule solaire du type hétérojonction silicium (type n)- $\operatorname{In}_{2} \mathrm{O}_{3}$ (dopé $\mathrm{Sn}$ ) est étudiée. Cette structure est préparée par une méthode de vaporisation simple et rapide. Les rendements de conversion obtenus sous éclairement solaire simulé AM1 sont voisins de $\eta=10 \%$. Les caractéristiques courant-tension et capacité-tension sont reportées en fonction de la température entre $T=77 \mathrm{~K}$ et $T=300 \mathrm{~K}$. L'ensemble des résultats est cohérent avec un modèle de jonction abrupte en présence d'une fine couche interfaciale isolante.
\end{abstract}

\begin{abstract}
The silicon (n-type)- $\operatorname{In}_{2} \mathrm{O}_{3}$ (Sn doped) heterojunction structure is studied. This structure is prepared using a very simple and fast spray method. Conversion efficiency up to $\eta=10 \%$ under AM1 simulated sunlight is reported. The current-voltage and capacitance-voltage characteristics are reported versus temperature between $T=77 \mathrm{~K}$ and $T=300 \mathrm{~K}$. The results agree with an abrupt junction model in presence of a thin insulating layer.
\end{abstract}

1. Introduction. - Des résultats récents $[1,2]$ ont montré qu'il était possible d'obtenir des rendements de conversion photoélectrique importants de l'ordre de 10 à $12 \%$ pour des hétérojonctions constituées par un dépôt d'oxyde d'indium dopé à l'étain, plus communément appelé I.T.O. (Indium Tin Oxide), sur un substrat de silicium monocristallin. L'intérêt de ce type de structure réside dans la plus grande facilité de préparation et par conséquent un coût plus faible que les diodes à jonction diffusées du type $\mathrm{p}-\mathrm{n}^{+}$généralement employées à l'heure actuelle.

Les films minces d'oxyde d'étain, d'oxyde d'indium ou les composés mixtes $\operatorname{In}_{2} \mathrm{O}_{3} / \mathrm{SnO}_{2}$ (I.T.O.) préparés par une méthode de vaporisation (spray) sont des semiconducteurs dégénérés de type $n\left(n \approx 10^{19}\right.$ à $10^{20} \mathrm{~cm}^{-3}$ ) ayant des largeurs de bande interdite comprises entre 3,5 et $4 \mathrm{eV}$ donc transparents sur toute l'étendue du spectre visible et proche infrarouge. Dans ces conditions, la couche d'I.T.O. joue le rôle d'une couche transparente, conductrice, protectrice et antiréfléchissante. En effet, leur indice est voisin de $n \approx 2$. Un tel film donne une réflexion nulle pour une longueur d'onde $\lambda_{0}=0,8 \mu \mathrm{m}$ lorsque $n_{\text {I. T.o. }}=\sqrt{n_{\mathrm{Si}}}$ et $d=\lambda_{0} / 4 n_{\text {I.T.o. }}, d$ étant son épaisseur, soit $d \approx 0,1 \mu \mathrm{m}$. La valeur de la résistivité de la couche I.T.O.,

$$
\rho_{\text {I.T.o. }} \approx 5 \times 10^{-4} \Omega . \mathrm{cm},
$$

(*) Associé au C.N.R.S. conduit, pour des épaisseurs aussi faibles, à des résistances séries importantes qui poseront des problèmes de collecte des porteurs photogénérés. A ce stade, il nous faut cependant noter que les paramètres de fabrication des lames minces I.T.O. sont en général déterminés afin d'obtenir un compromis entre la transparence et la résistance de surface $R_{\square}$. Dans ce but des études ont été faites et des facteurs de mérite définis $[3,4]$ correspondant à une optimisation. Le problème est cependant plus complexe : la transparence et la résistance de surface influent sur les valeurs du courant de court-circuit $I_{\text {sc }}$ ainsi que sur la valeur du facteur de remplissage, mais d'autres paramètres tels que les conditions de dépôt (principalement la température $T_{2}$ du substrat) ou le rapport atomique $\mathrm{Sn} / \mathrm{In}$ peuvent avoir une importance critique pour l'obtention d'une tension en circuit ouvert $V_{\text {oc }}$ importante.

Nous avons reporté précédemment [5] les résultats obtenus à partir de couches I.T.O. optimisées sur lame de verre en pyrex. Typiquement, on obtenait : $R_{\square} \approx 10 \Omega$, transmission $=0,8$ à 0,9 entre $\lambda=0,4$ et $1,5 \mu \mathrm{m}$, ceci pour des rapports atomiques $\mathrm{Sn} / \mathrm{In}=0,023$ et des températures de substrat $T_{2} \approx 500^{\circ} \mathrm{C}$. Les rendements de conversion obtenus sous AM1 étant voisins de $\eta=7 \%$ avec $J_{\mathrm{sc}} \approx 30 \mathrm{~mA} \mathrm{~cm}^{-2}$ et $V_{\mathrm{oc}} \approx 400 \mathrm{mV}$. Les résultats ont été améliorés ultérieurement en changeant les paramètres de fabrication de la couche $\operatorname{In}_{2} \mathrm{O}_{3}(\mathrm{Sn})$. En particulier la température $T_{2}$ a été abaissée à $T_{2} \approx 435^{\circ} \mathrm{C}$ et le rapport atomique 
$\mathrm{Sn} /$ In augmenté à 0,25 . Les résultats suivants sont alors obtenus : $R_{\square} \approx 400 \Omega$ et transmission $=0,8$ à 0,95 entre $\lambda=0,4$ et $\lambda=2 \mu \mathrm{m}$, ce qui correspond à un abaissement très important du facteur de mérite [3] de ces couches I.T.O., mais les rendements de conversion atteignent dans ce cas [1] $\eta=10 \%$ avec $J_{\mathrm{sc}} \approx 30 \mathrm{~mA} / \mathrm{cm}^{2}$ et $V_{\text {oc }} \approx 500 \mathrm{mV}$.

Dans la suite de cet article nous présenterons (sauf précisions contraires) les résultats obtenus concernant ce deuxième type de cellules.

2. Préparation de la structure. - La méthode de préparation utilisée a été décrite en détail par ailleurs $[6,7]$. Une solution alcoolique d'un mélange $\mathrm{SnCl}_{4}$ et $\mathrm{InCl}_{3}$ est vaporisée (en utilisant l'azote comme gaz porteur) à travers un four de préchauffage (température $T_{1}=560^{\circ} \mathrm{C}$ ) sur le substrat de silicium dont la température est $T_{2}=435^{\circ} \mathrm{C}$. Les spécifications des fours assurent une excellente uniformité tant pour l'épaisseur que pour les propriétés physiques du film mince I.T.O. La solution conduisant aux meilleurs résultats pour le rendement de conversion était constituée, en concentration pondérale, par :

$$
\mathrm{H}_{2} \mathrm{O}(0,4204) ; \quad \mathrm{CH}_{3} \mathrm{CH}_{2} \mathrm{OH}(0,4204) ;
$$

trichlorure d'indium $\operatorname{InCl}_{3}(0,06)$; tétrachlorure d'étain hydraté $\mathrm{SnCl}_{4}, 5 \mathrm{H}_{2} \mathrm{O}(0,024)$ et $\mathrm{HCl}(0,075)$. Typiquement, le temps de vaporisation étant compris entre 2 et $3 \mathrm{~min}$., on obtient pour un dép $̂$ t sur support pyrex : $R_{\square} \approx 200$ à $400 \Omega$, transmission $\approx 0,9$ entre $\lambda=0,4$ et $2 \mu \mathrm{m}$, le rapport atomique $\mathrm{Sn} / \mathrm{In}$ dans la couche [8] étant voisin de 0,25 . Nous avons utilisé pour l'élaboration de la structure Si-I.T.O. des substrats de silicium de type $n$, orientation $\langle 111\rangle$, résistivités comprises entre 1 et $8 \Omega$.cm. Ces substrats sont munis d'un contact ohmique obtenu par diffusion de phosphore à $T=1000^{\circ} \mathrm{C}$. Après un nettoyage chimique au $\mathrm{CP}_{4}$, le silicium est rincé dans $\mathrm{HF}(50 \%)$ pendant 1 minute. Lorsque le dépôt a été effectué, les surfaces des diodes sont délimitées par réduction de l'I.T.O. à l'hydrogène naissant (poudre $\mathrm{Zn}$ et $\mathrm{HCl}$ ). La structure est complétée par l'évaporation d'une grille en argent pour les diodes à grandes surfaces.

3. Résultats expérimentaux. - Les mécanismes de conduction sont analysés pour cette structure. Dans ce but, les caractéristiques courant-tension et capacitétension ont été étudiées à différentes températures entre $T=77 \mathrm{~K}$ et $300 \mathrm{~K}$ ainsi que la courbe de réponse spectrale à $T=300 \mathrm{~K}$. De nombreuses diodes ont été étudiées tant sur un même substrat que sur des substrats différents, la reproductibilité des résultats est en général bonne.

3.1 CARACTÉRISTIQUES COURANT-TENSION-TEMPÉRATURE. - Nous avons porté sur la figure 1 les caractéristiques courant-tension pour une structure $\mathrm{Si}(\mathrm{n})$ I.T.O., dont la résistivité du substrat de silicium

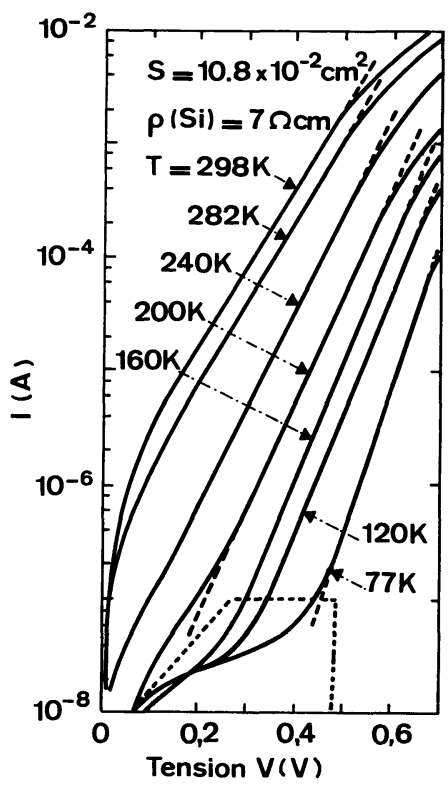

Fig. 1. - Caractéristiques courant-tension directes. Dans l'encadré en pointillé il n'a pas été possible de modéliser les caractéristiques $I-V$ par une loi du type éq. (3) du texte.

[Forward $I-V$ characteristic. The insert show parts of $I-V$ characteristics which do not follow eq. (3) in the text.]

est $\rho=7 \Omega . \mathrm{cm}$. Un circuit équivalent classique permet en général d'obtenir une loi de la forme :

$$
\begin{aligned}
J & =J_{\mathrm{s} 1}(T)\left[\exp \frac{q\left(V-J R_{\mathrm{s}}\right)}{k T}-1\right]+ \\
& +J_{\mathrm{S} 2}(T)\left[\exp \frac{q\left(V-J R_{\mathrm{s}}\right)}{n k T}-1\right]+\frac{V-J R_{\mathrm{s}}}{R_{\mathrm{sh}}}
\end{aligned}
$$

Le premier terme représente la contribution du courant classique associé à un contact métal-semiconducteur. Le deuxième terme représente la contribution du courant de génération-recombinaison dans la zone de charge d'espace $[9$, p. 647] et la valeur du paramètre $n$ est inférieure à 2 dans le cadre de ce modèle. $J_{\mathrm{S} 1}(T)$ et $J_{\mathrm{S} 2}(T)$ sont les courants de saturation respectifs qui sont fonction de la température et $R_{\mathrm{s}}$ et $R_{\mathrm{sh}}$ les résistances série et shunt.

Un deuxième modèle $[10,11]$ prenant en compte la présence d'une couche isolante à l'interface et caractéristique d'un mécanisme de conduction par effet tunnel assisté par des états d'interface, conduit à une relation $J-V$ :

$$
J=J_{\mathrm{S}}(T) \exp \left(\frac{V}{V_{0}}\right)
$$

$V_{0}$ étant un paramètre indépendant de $T$.

Nous avons tenté une modélisation de nos structures par utilisation d'un calculateur HP 9825 A associé à un traceur de courbe. Il n'a pas été possible d'adapter les caractéristiques $I-V-T$ expérimentales à une relation théorique du type équation (1) pour des valeurs de $n<2$. De même le modèle correspondant à 
l'équation (2) conduit à des valeurs de $V_{0}$ dépendant de $T$ comme cela est visible sur la figure 1 ( $V_{0}$ variant de $60 \mathrm{mV}$ à $298 \mathrm{~K}$ à $35 \mathrm{mV}$ à $77 \mathrm{~K}$ ). Nous avons alors utilisé la relation semi-empirique suivante :

$J=J_{\mathrm{s}}(T)\left[\exp \frac{q\left(V-J R_{\mathrm{s}}\right)}{n(T) k T}-1\right]+\frac{V-J R_{\mathrm{s}}}{R_{\mathrm{sh}}}$.

Dans le cadre de ce modèle nous avons obtenu un excellent accord jusqu'à la température $T=200 \mathrm{~K}$ sur toute la plage de courant étudié. Pour des températures inférieures à $200 \mathrm{~K}$ l'accord est excellent pour $I>10^{-7} \mathrm{~A}$, mais pour $I<10^{-7} \mathrm{~A}$ il n'est pas possible de trouver une valeur de $R_{\mathrm{sh}}$ dans l'équation (3) permettant de retrouver la partie encadrée de la courbe expérimentale, figure 1. Le tableau I résume les paramètres ainsi déterminés.

Tableau I. - Facteur de qualité $n, R_{\mathrm{s}}, R_{\mathrm{sh}}$ et $J_{\mathrm{S}}$ en fonction de la température T. Structure $\mathrm{Si}$ ( $n$, $\rho=7 \Omega . \mathrm{cm})-\operatorname{In}_{2} \mathrm{O}_{3}(\mathrm{Sn})$.

[Ideality factor $n, R_{\mathrm{S}}, R_{\mathrm{sh}}$ and $J_{\mathrm{S}} v s$. temperature $T$ $\mathrm{Si}(n, \rho=7 \Omega . \mathrm{cm})-\operatorname{In}_{2} \mathrm{O}_{3}(\mathrm{Sn})$ structure.]

$\begin{array}{ccc}T(\mathrm{~K}) & n & R_{\mathrm{s}}\left(\mathbf{\Omega} \cdot \mathrm{cm}^{2}\right) \\ - & - & - \\ 298 & 2,35 & 1,08 \\ 282 & 2,45 & 1,08 \\ 240 & 2,55 & 1,404 \\ 200 & 2,71 & 2,6 \\ 160 & 3,1 & 3,46 \\ 120 & 3,91 & 3,35 \\ 77 & 5,17 & 4,43\end{array}$

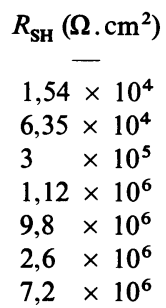

$$
\begin{gathered}
J_{\mathrm{S}}(T)\left(\mathrm{A} \cdot \mathrm{cm}^{-2}\right) \\
- \\
5,17 \times 10^{-6} \\
2,37 \times 10^{-6} \\
1,72 \times 10^{-7} \\
1,125 \times 10^{-8} \\
8,71 \times 10^{-10} \\
1,64 \times 10^{-10} \\
1,61 \times 10^{-12}
\end{gathered}
$$

En prenant pour dépendance de $J_{\mathrm{S}}$ en fonction de $T$ une variation de la forme

$$
J_{\mathrm{S}}(T)=A_{n}^{* *} T^{2} \exp \left(-\frac{\varphi_{B n}}{k T}\right)
$$

$A_{n}^{* *}$ étant la constante de Richardson effective $(9$, p. 389) on obtient, voir figure 2 , à partir de la région haute température :

$$
\varphi_{B n} \approx 0,33 \mathrm{eV} \text { et } A_{n}^{* *} \approx 10^{-5} \mathrm{~A} \cdot \mathrm{cm}^{-2} \mathrm{~K}^{-2} .
$$

Cette valeur de $\varphi_{\boldsymbol{B} \boldsymbol{n}}$ est beaucoup trop faible par comparaison avec les valeurs de $V_{\text {oc }}$ obtenues. Ce résultat est caractéristique d'un mécanisme de conduction plus complexe à l'interface métal-semiconducteur.

\subsection{CARACTÉRISTIQUes CAPACITÉ-TEMPÉRATURe. -} Les caractéristiques $C-V$ pour les mêmes diodes ont été mesurées à la fréquence de $1 \mathrm{MHz}$ et pour différentes températures. On observe. voir figure 3 . que la loi $C^{-2}-V$ est bien suivie (c'est le cas de toutes les structures étudiées). Nous avons vérifié que la pente correspond à la densité de porteurs du matériau de départ. soit $N_{\mathrm{D}}=7,8 \times 10^{14} \mathrm{~cm}^{-3}$.

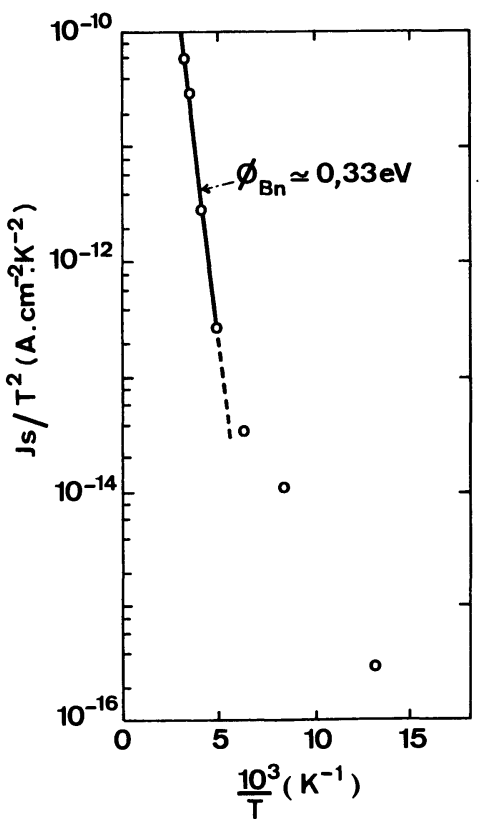

Fig. 2. - Energie d'activation, voir figure 1 .

[Activation energy plot, see figure 1.]

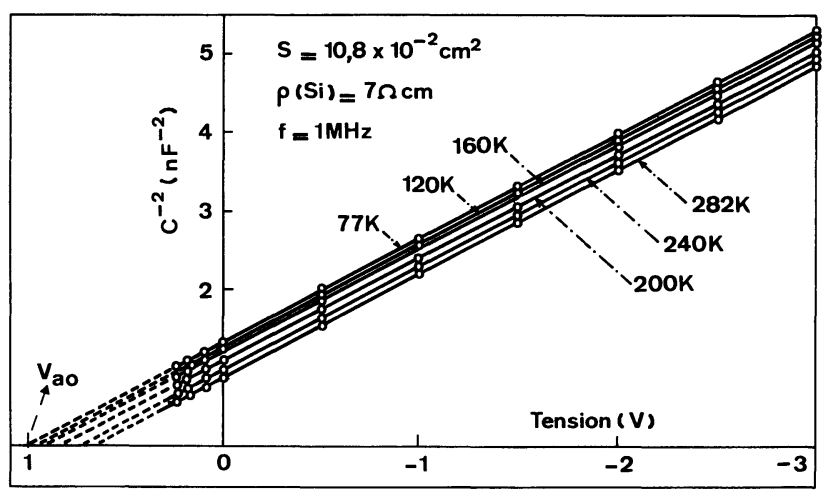

Fig. 3. - Caractéristiques capacité-tension.

[C-V characteristics.]

Les valeurs de $V_{\mathrm{a} 0}(T)$ obtenues par extrapolation pour $C^{-2}=0$ permettent d'en déduire la valeur de la hauteur de la barrière $\varphi_{B n}$ :

$$
\varphi_{B n}=V_{\mathrm{a} 0}+\frac{k T}{q}+\left(E_{\mathrm{c}}-E_{\mathrm{F}}\right)-f(\delta) .
$$

Dans cette expression le terme correctif $f(\delta)$ est une fonction de l'épaisseur $\delta$ de la couche isolante [12] à l'interface I.T.O.-silicium.

Le tableau II résume les principaux paramètres déterminés à partir de la figure 3. nous n'avons pas tenu compte de la contribution du terme en $f(\delta)$. On remarque que $\varphi_{B n}$ est sensiblement constant entre $282 \mathrm{~K}\left(\varphi_{B n} \approx 0,94 \mathrm{eV}\right)$ et $77 \mathrm{~K}\left(\varphi_{B n} \approx 1,08 \mathrm{eV}\right)$. Pour toutes les diodes préparées selon la méthode décrite nous avons trouvé $\varphi_{B n}=1 \pm 0.1 \mathrm{eV}$. On 
Tableau II. - $V_{\mathrm{a} o}, N_{\mathrm{D}}, \varphi_{\mathrm{Bn}}$ en fonction de la température $T$. Même diode que tableau $\mathrm{I}$.

$\left[V_{\mathrm{ao}}, N_{\mathrm{D}}, \varphi_{\mathrm{Bn}} v s\right.$. temperature $T$, same diode as in table I.]

$\begin{array}{cccc}T(\mathrm{~K}) & V_{\mathrm{ao}}(V) & N_{\mathrm{D}}\left(\mathrm{cm}^{-3}\right) & \varphi_{B n}(\mathrm{eV}) \\ - & - & - & - \\ 282 & 0,652 & 7,7 \times 10^{14} & 0,94 \\ 240 & 0,755 & 7,8 \times 10^{14} & 0,99 \\ 200 & 0,846 & 7,9 \times 10^{14} & 1,03 \\ 160 & 0,902 & 7,7 \times 10^{14} & 1,07 \\ 120 & 0,985 & 7,8 \times 10^{14} & 1,09 \\ 100 & 0,992 & 7,8 \times 10^{14} & 1,06 \\ 77 & 1,015 & 7,8 \times 10^{14} & 1,08\end{array}$

remarquera que la valeur ainsi déterminée pour $\varphi_{B n}$ est beaucoup plus importante que celle déduite de l'énergie d'activation des caractéristiques $I-V$. L'ensemble des résultats obtenus suggère fortement la présence d'une couche interfaciale, nous reviendrons sur ce point dans la discussion.

3.3 Mesures PhOtoÉlectriques. - La figure 4 représente les caractéristiques $I-V$ dans l'obscurité et sous éclairement solaire simulé AM1 pour deux substrats de silicium $\rho=2 \Omega$.cm et $\rho=7 \Omega$.cm. Les rendements de conversion obtenus sont voisins de $\eta=10 \%$ avec $V_{\text {oc }}$ variant de 470 à $500 \mathrm{mV}$ suivant les substrats (les conditions de préparation étant identiques), $J_{\text {sc }}$ varie de 28 à $32 \mathrm{~mA} . \mathrm{cm}^{-2}$ et le facteur de remplissage de 0,6 à 0,68 .

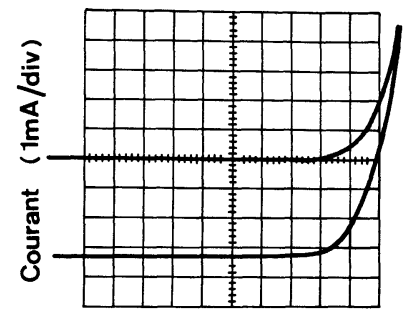

(a) Tension $(0.1 \mathrm{~V} / \mathrm{div})$

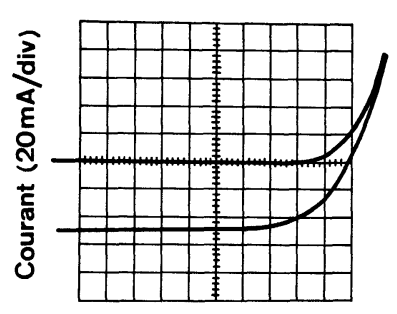

(b) Tension(0.1V/div)
Fig. 4. - Caractéristique $I-V$ dans l'obscurité et sous éclairement solaire simulé AM1. $T=296 \mathrm{~K}$. (a) $\mathrm{Si}(n, \rho=2 \Omega$.cm)-I.T.O., $S=0,1 \mathrm{~cm}^{2}$. (b) Si $\left(n, \rho=7 \Omega\right.$. cm)-I.T.O., $S=1,5 \mathrm{~cm}^{2}$.

[ $I-V$ characteristics in dark and under simulated sunlight AM1, $T=296$ K. (a) Si ( $n, \rho=2 \Omega$.cm)-I.T.O., $A=0.1 \mathrm{~cm}^{2}$. (b) Si (n, $\rho=7 \Omega$.cm)-I.T.O., $A=1.5 \mathrm{~cm}^{2}$.]

Nous avons observé une très légère sublinéarité dans la dépendance du photocourant $I_{\text {sc }}$ en fonction de la puissance lumineuse incidente. La tension en circuit est de la forme ([9], p. 643) :

$$
V_{\mathrm{oc}}=\frac{n K T}{q} \log \left(\frac{J_{\mathrm{sc}}}{J_{\mathrm{s}}}+1\right)
$$

avec une valeur de $n$ qui correspond sensiblement à celle déduite des caractéristiques $I-V$.
Ces résultats sont pratiquement identiques à ceux que nous avons obtenus pour des structures $\mathrm{Si}(\mathrm{n})$ $\mathrm{SnO}_{2}$ [13]. La courbe de réponse spectrale [1] indique un photocourant plus important vers les courtes longueurs d'onde que les diodes $\mathrm{p}-\mathrm{n}^{+}$classiques. La queue de réponse spectrale vers les grandes longueurs d'onde permet de déduire à partir de la relation :

$$
\sqrt{R} \simeq\left(h v-\varphi_{B n}\right)
$$

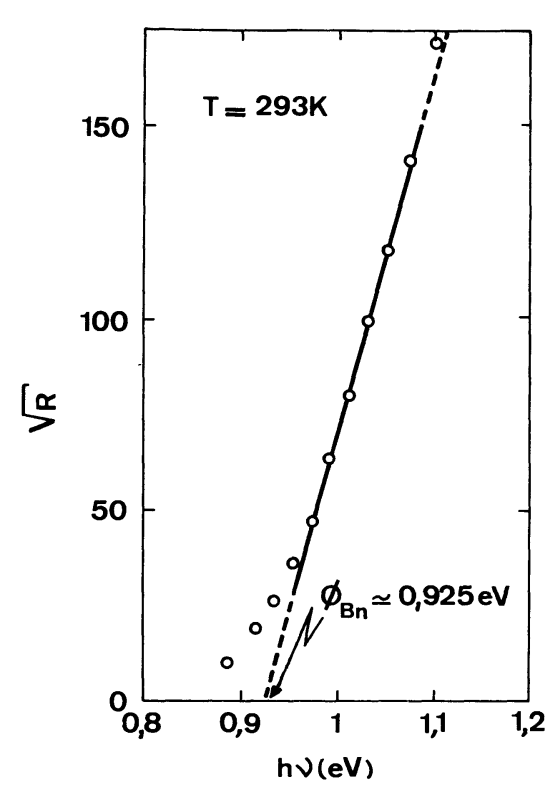

Fig. 5. - (Réponse spectrale) ${ }^{1 / 2}$ en fonction de l'énergie des photons incidents.

$\left[(\text { Spectral response })^{1 / 2}\right.$ vs. photon energy.]

la valeur de la hauteur de barrière (cf. Fig. 5). La valeur obtenue : $\varphi_{B n} \approx 0,925 \mathrm{eV}$ dans le cas de la figure 5 est en général plus faible de 0,03 à $0,05 \mathrm{eV}$ que celle déduite des mesures $C-V$. D'autre part, afin d'étudier les conséquences de la technique de préparation sur le substrat de silicium, nous avons évalué la longueur de diffusion des porteurs minoritaires $L_{\mathrm{p}}$ en utilisant deux méthodes de mesure (i) à partir de la réponse spectrale du courant de court-circuit $I_{\text {sc }}$ (ii) par des mesures de courant induit en utilisant un microscope à balayage JEOL (E.B.I.C.). La relation généralement vérifiée qui relie $I_{\mathrm{sc}}$ à $L_{\mathrm{p}}$ est [14] :

$$
I_{\mathrm{sc}}=(\mathrm{Cte}) \times L\left[\frac{\alpha L_{\mathrm{p}}}{1+\alpha L_{\mathrm{p}}}\right] .
$$

La mesure [15] consiste à ajuster la valeur de l'intensité lumineuse $L$ à différentes longueurs d'onde afin de conserver une valeur constante pour $I_{\text {sc }} \cdot \alpha$ est le coefficient d'absorption. On obtient ainsi :

$$
L(\lambda)=K\left(\alpha^{-1}(\lambda)+L_{\mathrm{p}}\right)
$$

où $K$ est une constante dépendant des caractéristiques de la cellule et de la valeur arbitraire choisie pour $I_{\text {sc }}$. 
La relation (9) est valable dans le cas où $L_{\mathrm{p}}$ est plus grande que la largeur $w$ de la zone de charge d'espace et inférieure à l'épaisseur $d$ du substrat de silicium. ( $d \approx 400 \mu \mathrm{m}$ dans le cas de nos cellules). La valeur du coefficient d'absorption $\alpha$ doit également vérifier les conditions : $\alpha w \ll 1$ et $\alpha d \gg 1$, correspondant à une énergie lumineuse absorbée dans la masse de l'échantillon.

La variation de $L$ en fonction de $\alpha^{-1}$ pour différentes longueurs d'onde nous a permis de déterminer par extrapolation $L_{\mathrm{p}} \approx 40-50 \mu \mathrm{m}$. La mesure de $L_{\mathrm{p}}$ par courant induit conduit à des valeurs plus faibles [1] $L_{\mathrm{p}} \approx 20-30 \mu \mathrm{m}$. Il faut remarquer cependant que par cette dernière méthode la contribution de la recombinaison en surface est importante, conduisant à une durée de vie effective des porteurs plus faible.

4. Discussion. - Le modèle d'une structure de type Schottky abrupte dans le silicium semble se confirmer. En effet, la reproductibilité des mesures $C-V-T$ est très bonne, les résultats obtenus pour différentes diodes prises sur le même substrat conduisent à des valeurs de $\varphi_{B n}$ identiques à $\pm 0,02 \mathrm{eV}$ près. La loi $C^{-2} \sim V$ est linéaire sur toute l'échelle de mesure (entre $+0,25 \mathrm{~V}$ et $-5 \mathrm{~V})$.

Pour différents substrats de silicium $n$ (les conditions de préparation étant identiques) ayant des résistivités entre 2 et $7 \Omega . \mathrm{cm}$, la dispersion dans les mesures de $\varphi_{B n}$ ne dépasse pas $0,05 \mathrm{eV}: \varphi_{B n}=0,9 \pm 0,05 \mathrm{eV}$ à $T=293 \mathrm{~K}$. Pour des jonctions abruptes, l'accord entre les valeurs de $\varphi_{B n}$ déterminées par mesures capacitives ou photoélectriques est en général excellent. Les résultats que nous avons obtenus conduisent cependant à une détermination plus faible d'environ $0,03 \mathrm{eV}$ pour la valeur de $\varphi_{B n}$ par une mesure photoélectrique. Il est probable que la présence d'une couche isolante interfaciale dont nous avons négligé l'influence (cf. relation (5), dans l'évaluation de $\varphi_{B n}(C-V)$ ), explique ce phénomène.

Dans le cadre de cette théorie [12] une épaisseur de 15 à $20 \AA$ suffit à expliquer cet écart.

Les caractéristiques $I-V$ - $T$ présentent par contre des variations importantes, aussi bien sur les valeurs de $J_{S}$ que de $n$. Les valeurs de $\varphi_{B n}(I-V)$ obtenues (en général $\varphi_{B n}(C-V)$ est égal à 3 ou 4 fois $\left.\varphi_{B n}(I-V)\right)$ associées aux très faibles valeurs de

$$
A_{n}^{* *} \approx 10^{-3} \text { à } 10^{-5} \mathrm{~A} \cdot \mathrm{cm}^{-2} \cdot \mathrm{K}^{-2}
$$

et aux valeurs importantes de $n>2$ à $T \approx 290 \mathrm{~K}$ confirment la présence d'une couche interfaciale isolante d'épaisseur non négligeable. Nous avons reporté figure 6 , la variation de $V_{\text {oc }} / n$ en fonction de $\varphi_{B n}(C-V)$ et $\varphi_{B n}(I-V)$ à $T=293 \mathrm{~K}$, pour des cellules obtenues par diverses conditions de préparation. On remarque que la loi idéale :

$$
V_{\mathrm{oc}} / n=\varphi_{B n}+k T / q \operatorname{In}\left[I_{\mathrm{sc}} /\left(A_{n}^{* *} \cdot T^{2}\right)\right]
$$

n'est pas suivie, la pente est positive mais différente de 1

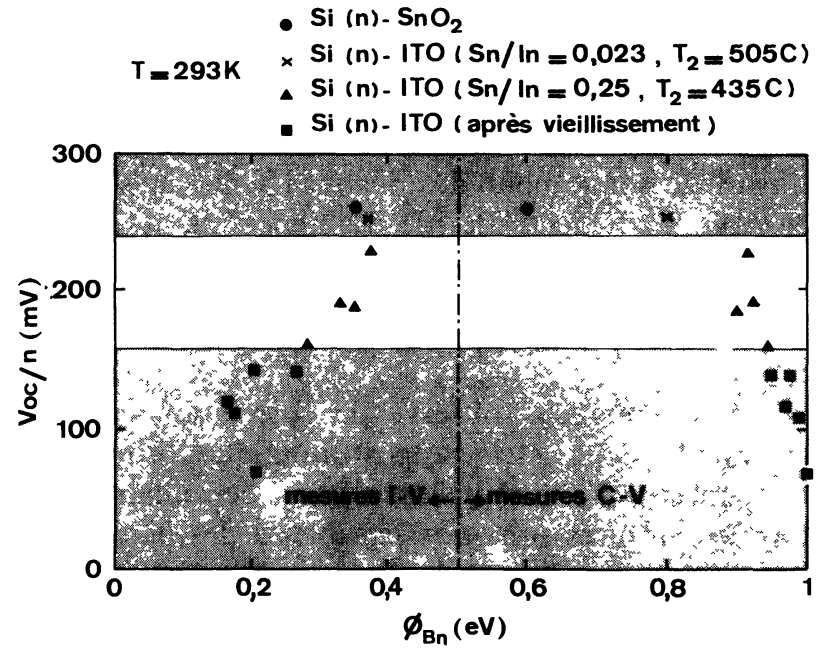

Fig. 6. $-V_{\text {oc }} / n$ en fonction de $\varphi_{B n}$ (mesures $C-V$ et $\left.I-V\right)$.

$\left[\left(V_{0}, / n\right)\right.$ vs. $\varphi_{B n}(C-V$ and $I-V$ measurements $\left.).\right]$

dans le cas de $V_{o} / n=f\left(\varphi_{B n}(I-V)\right)$ et elle est négative dans le cas de $V_{\mathrm{o}} / n=f\left(\varphi_{B n}(C-V)\right)$.

Nous avons observé cependant une corrélation entre la valeur de $V_{\text {oc }} / n$ et le rendement de conversion $\eta$. Les cellules analysées précédemment dans cet article ont des valeurs de $V_{\text {oc }} / n$ comprises entre 160 et $240 \mathrm{mV}$ environ, les valeurs de $n$ sont telles que $V_{\text {oc }} \approx 450$ à $500 \mathrm{mV}$ et le rendement de conversion sous AM1 varie entre 8 et $10 \%$, le facteur de remplissage est voisin de 0,65 . Les valeurs de $V_{\text {oc }} / n$ inférieures à $160 \mathrm{mV}$ correspondent simultanément à des valeurs de $n$ plus importantes, supérieures à 3 , et à des valeurs de $V_{\text {oc }}$ plus faibles. Les valeurs de $\varphi_{B n}$ déterminées par $C$ - $V$ sont voisines de $1 \mathrm{eV}$, la loi $C^{-2} \sim V$ étant toujours suivie. Le facteur de remplissage est faible de l'ordre de 0,5 . Le rendement de ces structures est inférieur à $7 \%$. Ce résultat pourrait être associé à une épaisseur de couche isolante à l'interface trop importante. Dans cette catégorie on trouve, voir figure 6 , des cellules dont le vieillissement s'est traduit par une baisse de rendement de conversion. Dans la dernière catégorie, les cellules pour lesquelles $V_{\text {oc }} / n>240 \mathrm{mV}$, les valeurs de $\varphi_{B n}(C-V)$ sont plus faibles $\varphi_{B n}<0,8 \mathrm{eV}$, de même les valeurs de $n$ sont inférieures à 1,5 . Il est probable que dans ce cas les faibles valeurs observées pour $V_{\text {oc }}$, donc pour le rendement de conversion, proviennent en partie d'une faible valeur de l'épaisseur $\delta$ de la couche interfaciale. Dans cette catégorie on trouve les structures Si-I.T.O. préparées à partir de solutions dont le rapport atomique $\mathrm{Sn} / \mathrm{In}$ est plus faible, voir figure 6, ainsi que les cellules $\mathrm{Si}-\mathrm{SnO}_{2}$. Le paramètre dépendant de manière critique des conditions de fabrication est la tension en circuit ouvert $V_{\text {oc }}$

Cette dispersion importante observée pour $V_{\text {oc }}$, dans le cas de substrats préparés en faisant varier les paramètres de fabrication peut s'expliquer soit par une variation de l'affinité électronique $\chi$ (i.e. travail de sortie) de l'I.T.O., soit par une variation de l'épaisseur $\delta$ de la couche interfaciale. Il semble qu'une 
variation de $\chi$ permettant de rendre compte de la variation de $V_{\text {oc }}$ soit à écarter.

En utilisant la méthode du condensateur vibrant de Lord Kelvin, nous avons effectué des mesures de travail de sortie pour des dépôts I.T.O. sur substrat en pyrex. L'électrode de référence étant en or, $W_{\mathrm{Au}}=4,75 \mathrm{eV}$, on évalue ainsi

$$
W_{\text {I.T.o. }} \approx \chi_{\text {I.T.O. }} \approx 4,95 \pm 0,15 \mathrm{eV} .
$$

Nous n'avons pas observé de corrélation entre la valeur de $W_{\text {I.T.o. }}$ et le rapport atomique $\mathrm{Sn} / \mathrm{In}$ ou la température $T_{2}$ du substrat. Il faut cependant noter que ces mesures sont très sensibles à des paramètres (tels que les conditions de nettoyage du dépôt I.T.O.) difficilement contrôlables. Des dépôts préparés dans ces conditions identiques présentent des écarts de l'ordre de $150 \mathrm{mV}$ dans la mesure de $W$. La deuxième hypothèse, faisant intervenir la présence d'une couche interfaciale isolante semble beaucoup plus probable. Il semble, par exemple, qu'un rapport atomique $\mathrm{Sn} / \mathrm{In}$ important, $\mathrm{Sn} / \mathrm{In}: 0,15$ à 0,25 , associé à une température $T_{2}$ du substrat plus faible, $T_{2}<450{ }^{\circ} \mathrm{C}$, favorise la croissance d'une couche d'oxyde conduisant aux rendements de conversion importants $\eta=10 \%$ observés. La présence de cette couche interfaciale dont les paramètres sont très difficiles à contrôler dans le cadre des méthodes de préparation utilisées peut expliquer les résultats contradictoires observés par différents auteurs $[1,2,16,17,18,19,20]$. Ces différents auteurs utilisent un schéma d'hétérojonction abrupte à l'interface Si-I.T.O. l'ensemble des caractéristiques $I-V$ et $C-V$ permettant d'évaluer la discontinuité $\Delta E_{\mathrm{c}}$ entre les bandes de conduction de l'I.T.O. et du silicium à l'interface. Les différentes valeurs obtenues sont : $\Delta E_{\mathrm{c}}=0 \mathrm{eV}$ [2]; 0,4 à $0,5 \mathrm{eV}[18] ; 0.6 \mathrm{eV}$ [20] et $1 \mathrm{eV}$ [1].

Nous avons également observé pour certains substrats une évolution de la valeur du rendement de conversion $\eta$ dans le temps. Ce vieillissement se traduisant par une dégradation de $V_{\text {oc }}$ associé à une augmentation $\varphi_{B n}(C-V)$ de la hauteur de barrière. Remarquons que ce vieillissement qui n'est pas la règle semble confirmer la réactivité de l'interface Si-I.T.O. et la croissance dans le temps de la couche isolante. Nous n'avons pas observé d'évolution sur une période d'un an dans les caractéristiques $I-V$ et $C$ - $V$ de la plupart des structures étudiées. le rendement de conversion est en général stable.

5. Conclusion et développement futur. - Nous avons préparé des structures Si-I.T.O. en utilisant une technique de vaporisation simple et rapide permettant d'obtenir des rendements de conversion sous AM1 $\eta=10 \%$. Il en résulte que cette structure ouvre des perspectives très intéressantes comme convertisseur photovoltaïque dans le cadre d'une utilisation commerciale.

Il nous semble pour l'instant peu probable que l'on puisse trouver un modèle théorique permettant d'expliquer dans le détail l'ensemble des résultats photoélectriques observés. De trop nombreux paramètres interviennent au cours de l'élaboration de la structure dont il est difficile d'évaluer avec précision l'influence sur les mécanismes de conduction. La dispersion des résultats obtenus par divers auteurs $[1,2,17,20]$ pour des structures identiques l'atteste.

Il ne fait donc pas de doute dans notre esprit que les efforts futurs doivent surtout être portés sur l'amélioration du rendement de conversion. Dans cette optique, des études sont en cours (mesures S.I.M.S.) qui devraient permettre d'obtenir des renseignements (épaisseurs, et composition) sur la couche isolante à l'interface Si-I.T.O. Un contrôle plus précis de cette couche interfaciale devrait permettre une amélioration sensible du rendement de conversion.

Remerciements. - Les auteurs remercient la D.G.R.S.T. pour son apport financier ainsi que M. J. Bonnet et les professeurs J. P. Fillard, L. Lassabatere et M. Savelli pour leur support.

\section{Bibliographie}

[1] Manifacier, J. C. et Szepessy, L., Appl. Phys. Lett. 31 (1977) 459.

[2] Dubow, J. B., Burk, D. E. et Sires, J. R., Appl. Phys. Lett. 29 (1976) 494.

[3] HaAcKe, G., J. Appl. Phys. 47 (1976) 4086.

[4] Iles, P. A. et Soclof, S. I., 12th Photovolt. Spec. Conf. Rec. (1976) p. 978, IEEE 1976.

[5] Szepessy, L., Manifacier, J. C., Wadi, K., Bresse, J. F. et SAVelli, M., I Col-Loqui International d'Energia Solar, Barcelona, Spain, 1977.

[6] Ishiguro, K., Sasaki, T., Arai, T. et Imai, I., J. Phys. Soc. Japan 13 (1958) 296.

[7] Manifacier, J. C., De Murcia, M. et Fillard, J. P., Mat Res. Bull. 10 (1975) 1215.

[8] Manifacier, J. C., Szepessy, L., Bresse, J. F., Perotin, M. et STuck, R., Mat. Res. Bull. à paraître.

[9] SzE, S. M., Physics of semiconductor devices (Wiley Interscience) 1969.

[10] Hovel, H. J., Semiconductors and semi-metals (Academic Press, New York) 1975, Vol. 11.
[11] Sites, J. R. et Dubow, J. B., Photovoltaic Solar Energy Conf., Luxembourg, p. 930, 1977 (D. Reidel publishing Cy, Dordrecht, Holland) 1978.

[12] Goodman, A. M., J. Appl. Phys. 34 (1963) 329.

[13] Perotin, M., Szepessy, L., Manifacier, J. C., Parot, P., Fillard, J. P. et Savelli, M., Int. Conf. on Solar Electricity, Toulouse, France, p. 48, 1976 (CNES, 31055 Toulouse Cedex).

[14] Rostron, R. W., IEEE Trans. Electron. Dev. 9 (1972) 1024.

[15] Stockes, E. D. et Chu, T. L., Appl. Phys. Lett. 30 (1977) 425.

[16] Fabre, E. et Tiburg, R., Int. Conf. on Solar Electricity, Toulouse, France, p. 493, 1976 (CNES, 31055 Toulouse Cedex).

[17] Anderson, R. L., Appl. Phys. Lett. 27 (1975) 691.

[18] Thompson, W. G. et Anderson, R. L., Solid State Electron. 21 (1978) 603.

[19] Nash, T. R. et Anderson, R. L., 12th Photovolt. Spec. Conf. Rec., p. 975, IEEE 1976.

[20] Mizrah, T. et Adler, D., Appl. Phys. Lett. 29 (1976) 682. 